\title{
Monitorando o cancelamento de procedimentos cirúrgicos: indicador de desempenho organizacional*
}

\author{
MONITORING CANCELLATIONS OF SURGICAL PROCEDURES: AN INDICATOR \\ OF ORGANIZATIONAL PERFORMANCE
}

MONITOREANDO EL CANCELAMIENTO DE PROCEDIMIENTOS QUIRÚRGICOS:
INDICADOR DEL DESEMPEÑO ORGANIZACIONAL

Márcia Galan Perroca1, Marli de Carvalho Jericón ${ }^{2}$, Solange Diná Facundin ${ }^{3}$

\section{RESUMO}

Este estudo, de natureza exploratória descritiva, tem como objetivo investigar a ocorrência e as causas de cancelamento de cirurgias programadas na unidade de Centro Cirúrgico em um hospital de ensino de capacidade extra no interior do estado de São Paulo. Os dados foram coletados durante três meses consecutivos em 2004, utilizando-se documentos institucionais e formulário elaborado pelas pesquisadoras. No período do estudo foram programadas 4.870 cirurgias na unidade de Centro Cirúrgico investigada, das quais 249 foram canceladas $(5,1 \%)$. As principais causas geradoras dos cancelamentos das cirurgias estavam relacionadas ao paciente $(57,8 \%)$, tais como, não comparecimento (56,3\%) e condição clínica desfavorável $(34,7 \%)$; à organização da unidade $(22,1 \%)$ devido à falta de leitos disponíveis para internação $(29,1 \%)$ e ocorrência de cirurgias de emergência (25,5\%); à alocação de recursos humanos $(17,7 \%)$ ou ainda à alocação de recursos materiais e equipamentos $(1,6 \%)$.

\section{DESCRITORES}

Procedimentos cirúrgicos operatórios.

Centro cirúrgico hospitalar. Hospitais de ensino.

\section{ABSTRACT}

This descriptive and exploratory study aims at investigating the occurrence and causes for the cancellations of scheduled surgeries in the Surgical Center unit of a large school hospital in the interior of the State of São Paulo. Data were collected during three consecutive months in 2004 using institutional documents and forms elaborated by the researchers. In the period of study, 4,870 surgeries were scheduled in the investigated unit, of which 249 were suspended, an average of $5.1 \%$. The main causes for the cancellations concerned the patients $(57.8 \%)$, such as his/her absence $(56.3 \%)$ and his/her unfavorable clinical conditions $(34.7 \%)$; the unit's organization $(22.1 \%)$, due to the lack of available beds for hospitalization $(29.1 \%)$ and the occurrence of emergency surgeries (25.5\%); and the allocation of human resources $(17.7 \%)$ or of material resources and equipment (1.6\%).

\section{KEY WORDS}

Surgical procedures,operative. Surgery departament, hospital. Hospitals, teaching.

\section{RESUMEN}

Este estudio, de naturaleza exploratoria descriptiva, tiene como objetivo investigar la ocurrencia y las causas de cancelación de cirugías programadas en la unidad de Centro Quirúrgico en un hospital de enseñanza de capacidad extra en el interior del estado de Sao Paulo. Los datos fueron recolectados durantes tres meses consecutivos en el 2004, utilizándose documentos institucionales y formulario elaborado por las investigadoras. En el período del estudio fueron programadas 4870 cirugías en la unidad de Centro Quirúrgico investigada, de las cuales 249 fueron canceladas $(5,1 \%)$. Las principales causas de cancelamiento de las cirugías estaban relacionadas al paciente $(57,8 \%)$, tales como, no asistencia $(56,3 \%)$ y condición clínica desfavorable $(34,7 \%)$; a la organización de la unidad $(22,1 \%)$ debido a la falta de camas disponibles para el internamiento $(29,1 \%)$ y ocurrencia de cirugías de emergencia $(25,5 \%)$; a la asignación de recursos humanos $(17,7 \%)$ o aun a la asignación de recursos materiales y equipamientos $(1,6 \%)$.

\section{DESCRIPTORES}

Procedimientos quirúrgicos operativos.

Servício de cirugia en hospital. Hospitales escuela.

\footnotetext{
Extraído do Projeto contemplado com a Bolsa de Auxílio ao Pesquisador (BAP) da Faculdade de Medicina de São José do Rio Preto (FAMERP). 1 Enfermeira. Doutora em Enfermagem. Docente do Departamento de Enfermagem Especializada da FAMERP.

marcia.perroca@ famerp.br

2 Enfermeira. Doutoranda. Docente do Departamento de Enfermagem Especializada da FAMER. marli.jerico@ famerp.br

3 Enfermeira. Docente do Departamento de Enfermagem Geral da FAMERP. solangefacundin@ ig.com.br
} 


\section{INTRODUÇÃO}

A melhoria contínua da qualidade da assistência e da eficiência do serviço oferecido à população, com racionalização de recursos, tem sido uma preocupação constante das instituições de cuidados de saúde. Para avaliação contínua da qualidade, produtividade e do impacto dos programas e serviços na saúde da população, as instituições utilizam instrumentos gerenciais denominados indicadores ${ }^{(1)}$. São medidas de desempenho, ou seja, instrumentos com foco no resultado esperado e processo essencial para a obtenção de resultados relacionados à qualidade do serviço. Os indicadores alertam quando ocorre desvio de uma situação considerada normal ou esperada sinalizando para que o processo em questão possa ser revisado, impedindo a instalação do problema ${ }^{(2)}$.

O movimento cirúrgico é considerado uma variável que interfere nos indicadores de qualidade e produtividade das instituições hospitalares. Constitui-se em parâmetros de avaliação de produtividade na sala de operação (SO); isto é, taxa de ocupação, tempo de permanência, recuperação anestésica, intervalo de tempo entre cirurgias, taxa de atraso e de suspensão de cirurgia ${ }^{(3)}$. Um dos requisitos para o processo de acreditação hospitalar, especificamente para a Unidade de Centro Cirúrgico (UCC), é a utilização de sistema de informação baseada em taxas e indicadores para obtenção de informação estatística e monitoramento de resultados ${ }^{(4)}$.

A problemática de cancelamento de cirurgias tem recebido atenção por parte de pesquisadores da área de saúde nas últimas décadas. Estudos sobre cancelamento de cirurgias eletivas, principalmente em hospitais públicos e universitários, apontam taxas que variam de $17,6 \%$ a $33 \%{ }^{(5-8)}$. A repercussão do cancelamento de cirurgia para o paciente e sua família é também objeto de investigação. O procedimento cirúrgico causa alterações na rotina de vida de uma pessoa, com conseqüente afastamento das atividades desenvolvidas junto ao trabalho, ao lar e necessidade de mobilização de recursos financeiros ${ }^{(7)}$, conseqüentemente, o seu cancelamento gera frustração ${ }^{(7-9)}$.

Estudos realizados para identificar as reações dos pacientes diante desta situação demonstraram sentimento de revolta, desconfiança na equipe, insegurança e intenso estresse $^{(5)}$; sentimentos negativos e de conformismo ${ }^{(9)}$; preocupação e falta de controle da situaçãa ${ }^{(10)}$. A comunicação do cancelamento cirúrgico não tem sido motivo de preocupação por parte da equipe de saúde ${ }^{(8)} \mathrm{e}$ da administração do hospital. A ocorrência deste evento é percebida como rotineira e inerente à estrutura funcional da instituição $0^{(7)}$. A explicação do cancelamento ao paciente, quando realizada, é incompleta e assistematizada ${ }^{(6)}$. Os motivos relatados nem sempre coincidem com os registrados pela equipe cirúrgica e pela equipe de enfermagem da unidade de internação ${ }^{(9)}$.
Embora muitos pesquisadores apontem o impacto do cancelamento de cirurgia sobre a instituição de saúde; uma escassa investigação sobre esta temática pode ser encontrada na literatura nacional. Tem sido documentada a inexistência de registro de cirurgias canceladas e de estatísticas das atividades realizadas em unidades de Centro Cirúrgico ${ }^{(5)}$. Justifica-se, dessa forma, a construção de indicador para avaliar o desempenho da unidade investigada no que se refere à ocorrência de cancelamento cirúrgico, a fim de se estabelecer medidas gerenciais corretivas.

Dessa forma, o presente estudo tem como objetivo investigar a ocorrência e as causas do cancelamento de cirurgias eletivas em um hospital de ensino.

\section{TRAJETÓRIA METODOLÓGICA}

\section{Delineamento da Pesquisa}

O estudo caracteriza-se como de natureza exploratória, descritiva, com abordagem quantitativa a ser realizado na Unidade de Centro Cirúrgico de um hospital escola de capacidade extra, localizado no interior do estado de São Paulo. Sua Unidade de Centro Cirúrgico é constituída por 25 salas de operações e realiza mensalmente, em média, 1600 cirurgias de pequeno, médio e grande porte nas diversas especialidades. Atende pacientes internados ou admitidos pelo ambulatório de cirurgia. A programação cirúrgica é desenvolvida no período das 7 às $19 \mathrm{~h}$, diariamente, de segunda a sexta-feira e aos sábados no período da manhã. Os períodos noturnos, finais de semana e feriados são destinados a casos de emergência. A casuística do estudo é constituída por todas as cirurgias de caráter eletivo canceladas no período de setembro a novembro de 2004.

\section{Instrumento e procedimento para coleta de informações}

O mapa contendo a programação cirúrgica mensal foi utilizado para verificar a ocorrência de cancelamento de cirurgias. Para levantamento das suas causas geradoras foi elaborado um formulário estruturado com dois agrupamentos de dados: 1- Características demográficas (idade, sexo, unidade de origem, tipo, porte e horário da cirurgia); 2 - Dados relativos às causas do cancelamento tais como classificação e momento em que ocorreram. As causas de cancelamento das cirurgias foram classificadas pelas autoras em intrínsecas ao paciente (condição clínica desfavorável, não comparecimento e outras), organizacionais e relacionadas à alocação de recursos humanos e materiais. A circunstância em que as cirurgias foram canceladas foram classificadas em antes e depois do preparo da sala de operação (SO) e durante o procedimento anestésico-cirúrgico.

A coleta de dados teve início após a autorização formal da instituição, da chefia de enfermagem da Unidade de Centro Cirúrgico e de orientação das circulantes de sala de ope- 
ração que atuavam nos turnos da manhã e da tarde sobre o preenchimento do instrumento a ser utilizado. Os formulários eram recolhidos diariamente pelas pesquisadoras e as informações complementadas.

\section{RESULTADOS}

Não houve diferença significativa em relação ao gênero dos pacientes que tiveram suas cirurgias suspensas (Tabela 1). Contudo, quando se considera a faixa etária, nota-se um predomínio de crianças $(12,5 \%)$ e de pacientes nas faixas etárias de 51 a $60(9,7 \%)$ e idosos, acima de 71 anos (9,2\%). Não foi possível identificar a idade de muitos pacientes $(\mathrm{n}=85)$, pois os dados estavam incompletos nos formulários de solicitação cirúrgica.

Tabela 1 - Dados demográficos dos pacientes que tiveram suas cirurgias suspensas ( $\mathrm{N}=249$ ) - São José do Rio Preto $-2004$

\begin{tabular}{lcc}
\hline \multicolumn{1}{c}{ VARIÁVEIS } & $\boldsymbol{N}$ & $\%$ \\
\hline $\begin{array}{l}\text { Sexo } \\
\text { masculino }\end{array}$ & 122 & \\
feminino & 127 & 59 \\
& & \\
Faixa etária (anos) & & \\
$\leq 10$ & 31 & 12,5 \\
& & \\
$11-20$ & 12 & 4,8 \\
$21-30$ & 14 & 5,6 \\
$31-40$ & 19 & 7,6 \\
$41-50$ & 20 & 8,0 \\
$51-60$ & 24 & 9,7 \\
$61-70$ & 21 & 8,4 \\
$\geq 71$ & 23 & 9,2 \\
Não identificados & 85 & 34,2 \\
\hline
\end{tabular}

A Tabela 2 apresenta as características das cirurgias canceladas, segundo planos de saúde dos pacientes, dias da semana e período de ocorrência, momento da suspensão e porte. A maior parte dos pacientes $(73,1 \%)$ eram usuários do Sistema Único de Saúde (SUS). Houve uma maior ocorrência de suspensão nas quartas feiras $(24,1 \%)$ no primeiro horário (7:00h) do turno da manhã (59\%). O segundo horário da tarde (16:00h) acusou a menor ocorrência de cancelamento de cirugias $(2,4 \%)$. Observa-se que os cancelamentos ocorreram, preponderantemente, antes do preparo e montagem da sala de operação $(76,3 \%)$ e apenas 4 deles $(1,6 \%)$ durante o procedimento anestésico-cirúrgico $(1,6 \%)$. No que se refere ao porte, as cirurgias de pequeno $(43,8 \%)$ e médio porte $(35,7 \%)$ foram as que apresentaram maior incidência de cancelamento
Tabela 2 - Características das cirurgias suspensas (N=249) São José do Rio Preto - 2004

\begin{tabular}{|c|c|c|}
\hline CARACTERÍSTICAS & $N$ & $\%$ \\
\hline \multicolumn{3}{|l|}{ Planos de Saúde } \\
\hline SUS & 182 & 73,1 \\
\hline Demais planos & 63 & 25,3 \\
\hline Particular & 4 & 1,6 \\
\hline \multicolumn{3}{|l|}{ Dias da semana } \\
\hline Segunda & 48 & 19,3 \\
\hline Terça & 38 & 15,2 \\
\hline Quarta & 60 & 24,1 \\
\hline Quinta & 47 & 18,9 \\
\hline Sexta & 48 & 19,3 \\
\hline Sábado & 8 & 3,2 \\
\hline \multicolumn{3}{|l|}{ Período } \\
\hline $1^{\circ}$ horário manhã & 147 & 59 \\
\hline $2^{\circ}$ horário manhã & 43 & 17,3 \\
\hline $1^{\circ}$ horário tarde & 53 & 21,3 \\
\hline $2^{\circ}$ horário tarde & 6 & 2,4 \\
\hline \multicolumn{3}{|l|}{ Momento da suspensão } \\
\hline Antes do preparo da SO & 190 & 76,3 \\
\hline Depois do preparo da SO & 55 & 22,1 \\
\hline $\begin{array}{l}\text { Durante procedimento } \\
\text { anestésico-cirúrgico }\end{array}$ & 4 & 1,6 \\
\hline \multicolumn{3}{|l|}{ Porte } \\
\hline Pequeno & 109 & 43,8 \\
\hline Médio & 89 & 35,7 \\
\hline Grande & 45 & 18,1 \\
\hline Não identificado & 6 & 2,4 \\
\hline
\end{tabular}

As principais causas geradoras dos cancelamentos das cirurgias (Tabela 3 ) estavam relacionadas ao paciente $(57,8 \%)$, tais como, não comparecimento $(56,3 \%)$ e condição clínica desfavorável $(34,7 \%)$. Problemas relacionados à organizacão da unidade $(22,1 \%)$, também ocasionaram uma parcela significativa de cancelamentos, principalmente relacionados à falta de leitos disponíveis para internação $(29,1 \%)$ e ocorrência de cirurgias de emergência $(25,5 \%)$. Foram também identificadas como causas de cancelamentos erros na programação cirúrgica $(18,2 \%)$, ou seja, excesso de agendamentos, paciente fictício, dentre outras. Cirurgias contaminadas realizadas anteriormente nas salas de operações responderam por 3,6\% dos cancelamentos.

Das 249 cirurgias suspensas, apenas 44 delas $(17,7 \%)$ tiveram como causa de suspensão fatores relacionados a alocacão de recursos humanos. Dentre eles, a mudança de conduta médica representou $43,2 \%$, enquanto que o tempo cirúrgico excedido $25 \%$. Há de se destacar, também, a falta de anestesiologista $(11,4 \%)$ e de cirurgião $(6,8 \%)$ bem como o cancelamento do procedimento pelo cirurgião $(6,8 \%)$. As causas relacionadas à alocação de recursos materiais e equipamentos representaram apenas 1,6\% do total das causas de suspensão. 
Tabela 3 - Causas de suspensão de cirurgias no estudo (N=249) - São José do Rio Preto - 2004

\begin{tabular}{|c|c|c|c|c|}
\hline \multirow[b]{2}{*}{ CAUSAS } & \multirow[b]{2}{*}{$\mathbf{n}$} & \multirow[b]{2}{*}{$\%$} & \multicolumn{2}{|c|}{ TOTAL } \\
\hline & & & $\mathbf{N}$ & $\%$ \\
\hline Relacionadas ao paciente & & & 144 & 57,8 \\
\hline Não comparecimento & 81 & 56,3 & & \\
\hline Condicão clínica desfavorável & 50 & 34,7 & & \\
\hline Falta de jejum & 8 & 5,5 & & \\
\hline Recusa à realizacão de cirurgia & 5 & 3,5 & & \\
\hline Relacionadas à organizacão da unidade & & & 55 & 22,1 \\
\hline Falta de vaga & 16 & 29,1 & & \\
\hline Cirurgia de emergência & 14 & 25,5 & & \\
\hline Erro na programação cirúrgica & 10 & 18,2 & & \\
\hline Falta de documentacão e exames & 6 & 10,9 & & \\
\hline Falta de comunicação & 4 & 7,3 & & \\
\hline Reserva de horário & 3 & 5,4 & & \\
\hline Cirurgia contaminada & 2 & 3,6 & & \\
\hline Relacionadas aos Recursos Humanos & & & 44 & 17,7 \\
\hline Mudanca de conduta médica & 19 & 43,2 & & \\
\hline Tempo cirurgico excedido & 11 & 25,0 & & \\
\hline Falta de anestesiologista & 5 & 11,4 & & \\
\hline Cancelamento pelo cirurgião & 3 & 6,8 & & \\
\hline Falta de cirurgião & 3 & 6,8 & & \\
\hline Falta de preparo pré-operatório & 2 & 4,5 & & \\
\hline Falta de avaliação clínica & 1 & 2,3 & & \\
\hline Relacionadas a Materiais e Equipamentos & & & 4 & 1,6 \\
\hline Falta de material & 3 & 75,0 & & \\
\hline Falta de equipamento & 1 & 25,0 & & \\
\hline Não identificadas & 2 & 100 & 2 & 0,8 \\
\hline TOTAL & & & 249 & 100,0 \\
\hline
\end{tabular}

Conforme se observa na Tabela 4, dentre as especialidades médicas que tiveram, com maior frequência, seus procedimentos cirúrgicos cancelados no período estudado, encontram-se a cirurgia otorrinolaringológica $(20,5 \%)$, seguida da cirurgia geral $(15,7 \%)$ e da cirurgia oftalmológica $(10,9 \%)$. O número de cirurgias suspensas nas especialidades otorrinolaringologia e cirurgia geral manteve-se elevado durante os meses de setembro (respectivamente $n=18$ e $n=16)$, outubro $(n=18$ e $n=12)$ e novembro $(n=15$ e $n=11)$. A cirurgia oftalmológica, contudo, apresentou um elevado número de suspensão apenas no mês de setembro $(n=18)$.

Tabela 4 - Taxa de suspensão de cirurgias por especialidade médica nos meses de setembro, outubro e novembro de 2004 (N=249) - São José do Rio Preto - 2004

\begin{tabular}{|c|c|c|c|c|c|c|c|c|c|}
\hline \multirow{2}{*}{$\begin{array}{l}\text { ESPECIALIDADES } \\
\text { CIRÚRGICAS }\end{array}$} & \multicolumn{2}{|c|}{ SETEMBRO } & \multicolumn{2}{|c|}{ OUTUBRO } & \multicolumn{2}{|c|}{ NOVEMBRO } & \multicolumn{3}{|c|}{ TOTAL } \\
\hline & Real & Susp & Real & Susp & Real & Susp & Real & Susp & $\%$ \\
\hline Ortopedia & 225 & 10 & 191 & 6 & 205 & 4 & 621 & 20 & 8,0 \\
\hline Oftalmologia & 203 & 18 & 173 & 7 & 180 & 2 & 556 & 27 & 10,9 \\
\hline Plástica & 194 & 5 & 189 & 8 & 184 & 9 & 567 & 22 & 8,8 \\
\hline Otorrino & 191 & 18 & 223 & 18 & 173 & 15 & 587 & 51 & 20,5 \\
\hline Cirurgia Geral & 168 & 16 & 153 & 12 & 167 & 11 & 488 & 39 & 15,7 \\
\hline Urologia & 139 & 10 & 117 & 5 & 110 & 3 & 366 & 18 & 7,3 \\
\hline Cirurgia Cardíaca & 120 & 6 & 126 & 2 & 147 & 3 & 393 & 11 & 4,4 \\
\hline Vascular & 82 & 4 & 58 & 5 & 66 & 4 & 206 & 13 & 5,2 \\
\hline Ginecologia & 77 & 6 & 63 & 2 & 82 & 5 & 222 & 13 & 5,2 \\
\hline Cirurgia Pediátrica & 62 & 9 & 53 & 4 & 42 & 1 & 157 & 14 & 5,6 \\
\hline Proctologia & 54 & 3 & 61 & 3 & 64 & 2 & 179 & 8 & 3,2 \\
\hline Neurologia & 50 & 2 & 66 & 3 & 61 & 1 & 177 & 6 & 2,4 \\
\hline Anestesiologia & 43 & 0 & 40 & 0 & 42 & 1 & 125 & 1 & 0,4 \\
\hline Gastronterologia & 32 & 0 & 26 & 1 & 29 & 3 & 87 & 4 & 1,6 \\
\hline Bucomaxila & 25 & 0 & 18 & 1 & 15 & 0 & 58 & 1 & 0,4 \\
\hline Cirurgia Torácica & 17 & 0 & 14 & 1 & 16 & 0 & 47 & 1 & 0,4 \\
\hline Outras * & 11 & 0 & 10 & 0 & 13 & 0 & 33 & 0 & 0 \\
\hline TOTAL & 1693 & 107 & 1581 & 78 & 1596 & 64 & 4870 & 249 & 100,00 \\
\hline
\end{tabular}

Real - cirurgias realizadas ; Susp - cirurgias suspensas ;

Outras* : transplante de medula óssea, cirurgia dermatológica, cirurgia hematológica 
No período do estudo foram programadas 4870 cirurgias na unidade de Centro Cirúrgico investigada, das quais 249 foram canceladas. $\mathrm{O}$ número de procedimentos cirúrgicos cancelados diminuiu gradualmente durante os meses: setembro $(n=107)$, outubro $(n=78)$ e novembro $(n=64)$. A taxa de cancelamento oscilou de $6,3 \%$ a $4,0 \%$, com uma média de $5,1 \%$ (Tabela 5).

Tabela 5 - Taxa mensal e geral de suspensão de cirurgias (N=249) - São José do Rio Preto - 2004

\begin{tabular}{lccc}
\hline \multicolumn{1}{c}{ CIRURGIAS } & SETEMBRO & OUTUBRO & NOVEMBRO \\
\hline Realizadas & 1693 & 1581 & 1596 \\
Suspensas & 107 & 78 & 64 \\
Taxa de Suspensão (\%) & 6,3 & 4,9 & 4,0 \\
\hline
\end{tabular}

\section{DISCUSSÃo}

Os resultados apontaram a quarta feira como o dia da semana com maior ocorrência de cancelamento cirúrgico $(24,1 \%)$. Na Unidade de Centro Cirúrgico investigada, as equipes cirúrgicas estão distribuídas nas diversas salas de operação em dias da semana e horários previamente acordados. Dessa forma, às quartas são realizadas, principalmente, cirurgias oftalmológicas e otorrinolaringológicas, em sua maioria de pequeno porte. A clientela da cirurgia oftalmológica constitui-se, predominantemente, de pacientes idosos, enquanto que as cirurgias ambulatoriais oftalmológicas de crianças. Esta distribuição explica os achados deste estudo, ou seja, a faixa etária com maior número de cirurgias canceladas, as cirurgias em sua maioria de pequeno porte $(43,8 \%)$ bem como a maior frequência de procedimentos cirúrgicos suspensos nas especialidades de cirurgia otorrinolaringológica $(20,5 \%)$, cirurgia geral $(15,7 \%)$ e cirurgia oftalmológica $(10,9 \%)$. Um estudo realizado anteriormente em hospital escola na cidade de São Paulo(7) encontrou igualmente elevadas taxas de suspensão entre as especialidades de cirurgia geral, oftalmologia e otorrinolaringologia, dentre outras.

As causas que mais contribuíram para a ocorrência de cancelamentos de cirurgias estavam relacionadas ao paciente $(57,8 \%)$, à organização da unidade $(22,1 \%)$, à alocação de recursos humanos $(17,7 \%)$ ou ainda à alocação de recursos materiais e equipamentos $(1,6 \%)$. Resultados semelhantes foram encontrados em pesquisa realizada em hospital público na região metropolitana de Fortaleza, ou seja, cancelamento de cirurgias programadas devido a eventos relacionados ao paciente $(52 \%)$, às questões organizacionais (15\%), aos recursos humanos (13\%) e a materiais e equipamentos $0,9 \%^{(7)}$. Outros estudos ${ }^{(11-12)}$ têm, igualmente, mostrado que as causas de suspensão de cirugia estão fortemente atreladas a fatores intrínsecos ao paciente.

O não comparecimento do paciente à cirurgia agendada (56,3\%) e condição clínica desfavorável (34,7\%) emergiram, nesta investigação, como principais causas geradoras dos cancelamentos das cirurgias relacionadas ao paciente. $\mathrm{Na}$ instituição estudada, as cirurgias são agendadas durante consulta ambulatorial, quando ocorre a avaliação clínica do paciente. Contudo, como os agendamentos são realizados com muita antecedência, o paciente está susceptível às mudanças em suas condições clínicas o que pode inviabilizar o procedimento cirúrgico. Outro aspecto a ser considerado é que muitos dos pacientes são crianças e idosos moradores em cidades adjacentes ao hospital, dependentes de transporte de seus municípios de origem para deslocamento. Acreditamos que tanto a avaliação e a orientação pré-operatória mais efetivas ao paciente cirúrgico poderiam reduzir os valores encontrados.

O absenteísmo do paciente à cirurgia programada foi aspecto destacado em estudo realizado em um hospital universitário, no município de São Paulo ${ }^{(8)}$. Neste, a frequência de cirurgias canceladas pelo não compararecimento do paciente representou $54,3 \%$ do total das cirurgias canceladas, resultado semelhante ao encontrado nesta pesquisa $(56,3 \%)$. A autora do estudo citado anteriormente também classificou os motivos para o absenteísmo do paciente em condição institucional, clínica, social e pessoal. O desconhecimento e alteração da data da cirugia, dificuldade de internação, falta de vaga e falta de exame préoperatório relacionam-se à condição institucional e representaram $53,3 \%$ do total dos não comparecimentos. A condição clínica desfavorável, tais como, infecção de vias aéreas superiores e outras infecções foi responsável por $28,3 \%$ dos não comparecimentos. Em estudo sobre suspensão de cirurgia de catarata em um hospital universitário $^{(12)}$, as causas relacionadas ao não comparecimento do paciente representaram $41 \%$ do total das cirurgias canceladas; enquanto que as causas relacionadas à condição clínica do paciente foram responsáveis pela suspensão de $4,5 \%$ dos procedimentos cirúrgicos. Em estudo anterior realizado no mesmo hospital ${ }^{(11)}$ foram encontrados valores de $12,6 \%$ para eventos relacionados à condição clínica desfavorável do paciente. $\mathrm{O}$ autor atribui esta redução à implantação no serviço de um grupo de orientação préoperatória. 
Problemas relacionados à organização da unidade também ocasionaram uma parcela significativa de cancelamentos $(22,1 \%)$, principalmente relacionados à falta de leitos disponíveis para internacão $(29,1 \%)$. Valores de $7 \%$ para suspensão de cirugias (oftalmológicas) por limitação de vagas têm sido relatados na literatura ${ }^{(12)}$. Em muitos hospitais, constitui-se regra básica que o paciente não seja admitido na Unidade de Centro Cirúrgico para cirurgias programadas sem a efetiva comprovação de vaga. Se a admissão do paciente na UCC estivesse vinculada à existência de vaga, acreditamos que haveria acentuada redução de cancelamentos de cirurgia na instituicão.

Outro aspecto que merece destaque foram os cancelamentos originados por erros na programacão cirúrgica $(18,2 \%)$, ou seja, excesso de agendamentos, paciente fictício, dentre outros. Por se tratar de hospital escola onde os acadêmicos ainda estão em processo de desenvolvimento de suas habilidades técnicas e profissionais, torna-se comum o agendamento com maior número de procedimentos cirúrgicos do que a especialidade possa realizar. Apesar de cada especialidade ter seu dia da semana, horário e sala de operação previamente estabelecidos, as equipes necessitam agendar a cirurgia até às 15 horas do dia anterior para garantirem a efetiva posse da sala. Muitas vezes, os acadêmicos criam pacientes fictícios para garantir a sala. A informatização do sistema e intercomunicação com as unidades de internação vêm reduzindo esta ocorrência indesejável. A elevada ocorrência de cancelamentos antes do preparo e montagem da sala de operação $(76,3 \%)$ evidencia também problemas na organização da unidade e erros de programação. A característica de hospital de ensino explica também o elevado número de cirurgias suspensas por mudança de conduta médica $(43,2 \%)$ e tempo cirúrgico excedido $(25 \%)$.

Causou-nos surpresa constatar que duas cirurgias $(3,6 \%)$ foram suspensas tendo como causa cirurgias contaminadas realizadas anteriormente nas salas de operações. Os conceitos mais atuais de limpeza de sala de cirurgia recomendam considerar todo procedimento cirúrgico como contaminado, ou seja, limpar com o mesmo rigor as salas após qualquer tipo de cirurgia, seja ela contaminada ou não ${ }^{(13)}$, bem como medidas-padrão de precauções se houver respingos de sangue ou fluidos corpóreos ${ }^{(14)}$.

No período de três meses foram programadas $4.870 \mathrm{ci}-$ rurgias na unidade de Centro Cirúrgico investigada, das quais 249 foram suspensas correspondendo a uma média de $5,1 \%$. Estudos sobre cancelamento de cirurgias eletivas, principalmente em hospitais públicos e universitários, apontam taxas de suspensão de 17,6\% (hospitais do nordeste) $)^{(5)}$, $33 \%$ na região metropolitana de Fortaleza $^{(6)}$ e $19,91 \%$ em hospital universitário na cidade de São $\mathrm{Paulo}^{(7)}$. No que concerne às cirurgias ambulatoriais, as taxas de suspensão variaram de $19,5 \%$ (cirurgia de facectomia) ${ }^{(12)}$ a $22,8 \%$ (cirurgias oftalmológicas) ${ }^{(11)}$. O maior número de investigações sobre ocorrência de cancelamento de cirurgia realizadas por instituições públicas e de ensino, remete-nos à reflexão se este evento é mais comum neste tipo de instituição devido à suas peculiaridades.

Os achados desta investigação evidenciam que grande parte dos fatores causadores poderiam ter sido evitados e previstos. O grande desafio não consiste apenas em se obter bons indicadores, mas sim em mantê-los e aprimorálos. Portanto, recomenda-se aos enfermeiros gerentes da unidade de Centro Cirúrgico redesenhar os processos que estão ligados diretamente a temática (visita pré-operatória, planejamento do mapa cirúrgico, recursos humanos, gerenciamento de materiais) e a utilização de método de controle para padronizar ou corrigir os problemas, ou seja, estabelecer medidas para reduzir as causas de suspensão cirúrgica traçando e perseguindo metas a serem atingidas. A utilização da capacidade máxima cirúrgica constitui uma das principais medidas que visam a eficiência, visto que pacientes cirúrgicos representam a maior receita hospitalar em uma instituição de cuidados de saúde.

\section{CONCLUSÃO}

São várias as investigações sobre a assistência ao paciente cirúrgico, mas os reflexos desta temática para as instituições hospitalares e, especificamente, a busca por indicadores que demonstrem a contribuição das funções administrativas do enfermeiro ainda é considerada um desafio. Para que os indicadores possam ser utilizados como apoio no processo de tomada de decisão eles precisam ser construídos de forma consistente ${ }^{(15)}$. Isto demanda infra-estrutura de apoio por meio de processos sistematizados para obtenção de dados confiáveis, geração de informações e de sensibilidade para o cuidado de enfermagem. Assim, o enfermeiro com habilidade na utilização de informações pode transformar o gerenciamento da UCC. Mostrará resultados positivos em relação à assistência prestada e à implementação de ações de melhoria baseadas em altos padrões de qualidade.

Esta pesquisa nos possibilitou uma análise situacional da unidade de centro cirúrgico por meio da identificação do indicador de desempenho de cancelamento cirúrgico. Estes dados contribuíram para o fluxo de informações, assim, permitiu aos profissionais avaliar e analisar aspectos importantes da assistência prestada. Sobretudo, possibilitando-lhes identificar problemas, direcionar programas de educação em serviço e melhorar o desempenho, adequando o processo de trabalho às necessidades do cliente.

Dessa forma, as autoras recomendam novas investigações com relação a esta temática para maior aprofundamento da discussão. Espera-se que os resultados encontrados possam auxiliar os enfermeiros no desenvolvimento de indicadores e outros que norteiem sua prática e os direcionem para resultados efetivos. 


\section{REFERÊNCIAS}

1. Bittar OJ, Nogueira V. Hospital: qualidade \& produtividade. São Paulo: Sarvier; 1996.

2. Knobel E. Condutas no paciente grave. $2^{a}$ ed. São Paulo: Atheneu; 2002.

3. Gatto MAF, Jouclas VMG. Otimizando o uso da SO. Rev SOBECC. 1998;3(1):23-8.

4. Brasil. Ministério da Saúde. Secretaria de Assistência à Saúde. Manual brasileiro de acreditação hospitalar. $3^{\mathrm{a}}$ ed. rev. atual. Brasília; 2002.

5. Vieira MJ. Causas e consequiências da suspensão de cirurgias: estudo exploratório em uma cidade do nordeste do país [dissertação]. Rio de Janeiro: Escola de Enfermagem, Universidade Federal do Rio de Janeiro; 1982.

6. Yokota O. A problemática cancelamento de cirurgia conforme percebida e expressada pelos pacientes: um estudo exploratório em um hospital-escola do norte do Paraná [dissertação]. Ribeirão Preto: Escola de Enfermagem de Ribeirão Preto, Universidade de São Paulo; 1983.

7. Cavalcanti JB, Pagliuca LMF, Almeida PC. Cancelamento de cirurgias programadas em um hospital-escola: um estudo exploratório. Rev Lat Am Enferm. 2000;8(4):59-65.

8. Paschoal MLH. Taxa de suspensão de cirurgia em um hospital universitário e os motivos de absenteísmo do paciente à cirurgia programada [dissertação]. São Paulo: Escola de Enfermagem, Universidade de São Paulo; 2002.
9. Bianchi ERF. Estudo exploratório sobre suspensão de cirurgia: a comunicação da suspensão e a reação relatada pelo paciente frente ao fato [dissertação]. São Paulo: Escola de Enfermagem, Universidade de São Paulo; 1983.

10. Antonio PS, Munari DB, Costa HK. Fatores geradores de sentimentos do paciente internado frente ao cancelamento de cirurgias. Rev Eletr Enferm [periódico na Internet]. 2002 [citado 2005 out. 15];4(1):33-9. Disponível em://http:// www.fen.ufg.br.

11. Arieta CEL, Taiar A, Kara-José N. Utilização e causas de suspensão de intervenções cirúrgicas oculares em Centro Cirúrgico ambulatorial universitário. Rev Assoc Med Bras. 1995; 41(3):233-5.

12. Lira RPC, Nascimento MA, Temporini ER, Kara-José N, Arieta CEL. Suspensão de cirurgias de catarata e suas causas. Rev Saúde Pública. 2001;35(5):487-9.

13. Association of Operation Room Nurses (AORN). Práticas recomendadas para precauções-padrão baseadas em transmissão de patógenos no perioperatório. Rev SOBECC. 1999; 4(4):12-5.

14. Brasil. Ministério da Saúde. Processamento de artigos e superfícies em estabelecimentos de saúde. Brasília; 1994.

15. Cianciarullo IT, Cornetta VK. Saúde, desenvolvimento e globalização: um desafio para os gestores do terceiro milênio. São Paulo: Ícone; 2002. 